CALT-68-1783

UCSD/PTH 92-17

DOE RESEARCH AND

DEVELOPMENT REPORT

\title{
Baryons Containing a Heavy Quark as Solitons ${ }^{\star}$ (REVISED VERSION)
}

\author{
Elizabeth Jenkins and Aneesh V. Manohar \\ Department of Physics, University of California, San Diego, \\ 9500 Gilman Drive, La Jolla, CA 92093-0319 \\ Mark B. Wise \\ California Institute of Technology, Pasadena, CA 91125
}

\begin{abstract}
The possibility of interpreting baryons containing a single heavy quark as bound states of solitons (that arise in the nonlinear sigma model) and heavy mesons is explored. Particular attention is paid to the parity of the bound states and to the role of heavy quark symmetry.
\end{abstract}

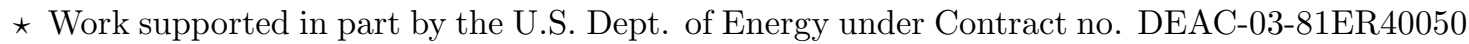
and under grant no. DE-FG03-90ER40546, and by the National Science Foundation under a Presidential Young Investigator award no. PHY-8958081. 
Many properties of baryons $B$ containing light $u$ and $d$ quarks suggest that they can be represented as solitons. For example, they have a mass of order $1 / \alpha$ and the cross section for $e^{+} e^{-} \rightarrow B \bar{B}$ is of order $e^{-1 / \alpha}$, where $\alpha=1 / N_{c}$ is taken as a small quantity. ${ }^{1}$ Skyrme originally suggested that baryons are solitons in the chiral Lagrangian (i.e. nonlinear sigma model) used to describe pion self interactions. ${ }^{2}$ The solitons of the chiral Lagrangian have the right quantum numbers to be QCD baryons, ${ }^{3}$ provided one includes the Wess-Zumino term. ${ }^{4}$ The model of QCD baryons as solitons has been used to compute many of their properties. ${ }^{5,6}$ The same large $N_{c}$ power counting that suggests that baryons containing only light quarks are solitons, also suggests that baryons containing a single heavy quark $Q\left(m_{Q} \gg \Lambda_{Q C D}\right)$ can be represented as solitons.

Recently there has been considerable progress in understanding the properties of hadrons containing a single heavy quark. In the limit $m_{Q} \rightarrow \infty$, QCD has a heavy quark spin-flavor symmetry ${ }^{7,8}$ that determines many properties of hadrons containing a single heavy quark. In the $m_{Q} \rightarrow \infty$ limit, the total angular momentum of the light degrees of freedom (i.e. light quarks and gluons),

$$
\vec{S}_{\ell}=\vec{S}-\vec{S}_{Q},
$$

is conserved. Consequently mesons and baryons containing a single heavy quark can be labeled by $s_{\ell}$, and provided $s_{\ell} \neq 0$, they come in degenerate doublets ${ }^{9}$ with total spins

$$
s_{ \pm}=s_{\ell} \pm 1 / 2
$$

formed by combining the spin of the heavy quark with the angular momentum of the light degrees of freedom.

Callan and Klebanov originally suggested an interpretation of baryons containing a heavy quark as bound states of solitons of the pion chiral Lagrangian with mesons containing a heavy quark. ${ }^{10,11}$ In this paper we examine this model for heavy baryons. An important aspect of this work is that the consequences of heavy quark symmetry 
are taken into account. Heavy quark symmetry implies that a doublet of mesons containing the heavy quark must be considered and that both members of the doublet can occur in the bound state. In this regard the work presented here differs from that of Callan and Klebanov. An important feature of the work of Callan and Klebanov was that the ground state of the heavy baryon is in an $L=1$ partial wave, and thus the lowest mass baryon containing a heavy quark has positive parity in accord with experiment. In this paper both the soliton and meson are taken as infinitely heavy and it is assumed that the soliton-meson potential is minimized at the origin. Consequently, the spatial wavefunction for the ground state is a Dirac delta function $\delta^{3}(\vec{x})$ and corresponds to the lowest energy classical configuration which has the soliton and heavy meson located at the same spatial point. We give a simple explanation of the Callan-Klebanov parity flip appropriate to this description of the bound state.

The lowest lying heavy mesons with $Q \bar{q}_{a}\left(q_{1}=u, q_{2}=d\right)$ flavor quantum numbers have $s_{\ell}=1 / 2$ and come in a doublet containing spin-zero and spin-one mesons. For $Q=c$ these are the $D$ and $D^{*}$ mesons. The interactions of these heavy mesons with pions are described by a chiral Lagrangian which is invariant under both heavy quark spin symmetry and chiral $S U(2)_{L} \times S U(2)_{R}$ symmetry. Chiral $S U(2)_{L} \times S U(2)_{R}$ symmetry is spontaneously broken to the vector $S U(2)_{V}$ subgroup, so the Goldstone boson manifold is $S U(2)_{L} \times S U(2)_{R} / S U(2)_{V}$. A general Goldstone boson field configuration is obtained by making a space-time dependent chiral transformation $\left(g_{L}(x), g_{R}(x)\right)$ on a standard vacuum state. The Goldstone boson fields $\Xi(x)$ in a $G / H$ sigma model are defined by ${ }^{12}$

$$
g(x)=\Xi(x) h(x)
$$

where $g(x) \in G$ describes the locally rotated vacuum, $h(x) \in H$, and $\Xi(x)$ is generated by $n_{g}$ independent broken generators, where $n_{g}$ is the number of Goldstone bosons.

$\star$ We will restrict the analysis to the case of two light flavors; the generalization to three flavors is straightforward. 
Different choices for the $n_{g}$ broken generators lead to different (but equivalent) nonlinear realizations of the spontaneously broken chiral symmetry. The standard $\Sigma$ basis for the QCD chiral Lagrangian is defined by

$$
\left(g_{L}(x), g_{R}(x)\right) \equiv(\Sigma(x), 1) \cdot\left(V_{\Sigma}(x), V_{\Sigma}(x)\right)=\left(\Sigma(x) V_{\Sigma}(x), V_{\Sigma}(x)\right)
$$

where the broken generators are chosen to be the $S U(2)_{L}$ generators $T_{L}^{A}$. The field $\Sigma(x)$ is equal to $g_{L}(x) g_{R}^{\dagger}(x)$, and transforms under chiral $S U(2)_{L} \times S U(2)_{R}$ as

$$
\Sigma(x) \rightarrow L \Sigma(x) R^{\dagger}
$$

since $g_{L}(x) \rightarrow L g_{L}(x), g_{R}(x) \rightarrow R g_{R}(x)$. The pion fields occur in the $2 \times 2$ matrix $M$,

$$
M=\left[\begin{array}{cc}
\pi^{0} / \sqrt{2} & \pi^{+} \\
\pi^{-} & -\pi^{0} / \sqrt{2}
\end{array}\right],
$$

where

$$
\Sigma=\exp \left(\frac{2 i M}{f}\right)
$$

and $f$ is the pion decay constant. Experimentally $f \simeq 132 \mathrm{MeV}$. In the large $N_{c}$ limit $f \sim 1 / \sqrt{\alpha}$ (recall that $\alpha=1 / N_{c}$ ). For considering the couplings of heavy mesons to the pseudo-Goldstone bosons, it is useful to introduce the $\xi$ basis defined by

$$
\left(g_{L}(x), g_{R}(x)\right) \equiv\left(\xi(x), \xi^{\dagger}(x)\right) \cdot\left(V_{\xi}(x), V_{\xi}(x)\right)=\left(\xi(x) V_{\xi}(x), \xi^{\dagger}(x) V_{\xi}(x)\right)
$$

where the broken generators are chosen to be the axial generators $T_{L}^{A}-T_{R}^{A}$. Under chiral $S U(2)_{L} \times S U(2)_{R}, \xi$ transforms as

$$
\xi(x) \rightarrow L \xi(x) U^{\dagger}(x)=U(x) \xi(x) R^{\dagger}
$$

where $U$ is a complicated function of $L, R$ and the pion fields and in general depends on the space-time coordinates. However, for an unbroken $S U(2)_{V}$ transformation, 
$V=L=R, U(x)$ is equal to $V$, and is constant. Comparing eq. (8) with eq. (4), we see that $\xi$ and $\Sigma$ are related by

$$
\Sigma(x)=\xi^{2}(x)
$$

The Goldstone boson manifold $S U(2)_{L} \times S U(2)_{R} / S U(2)_{V}$ is diffeomorphic to the group manifold $S U(2)$, which is diffeomorphic to the three-sphere $S^{3}$. The standard identification of $\Sigma$ with $S^{3}$ is obtained by writing

$$
\Sigma=a+i \vec{b} \cdot \vec{\tau}, \quad a^{2}+|\vec{b}|^{2}=1
$$

and identifying $\Sigma$ with the point $(a, \vec{b}) \in S^{3}$. The $\Sigma$ basis provides a single-valued coordinate system that covers the entire surface of $S U(2)$, because

$$
\left(g_{L}, g_{R}\right)=\left(\Sigma V_{\Sigma}, V_{\Sigma}\right)=\left(\Sigma^{\prime} V_{\Sigma}^{\prime}, V_{\Sigma}^{\prime}\right)
$$

implies that $\Sigma=\Sigma^{\prime}$.

Unlike the $\Sigma$ basis, the $\xi$ basis is not a well-defined coordinate system on $S U(2)$. Two fields $\xi$ and $\xi^{\prime}$ denote the same Goldstone boson configuration if

$$
\left(g_{L}, g_{R}\right)=\left(\xi(x) V_{\xi}(x), \xi^{\dagger}(x) V_{\xi}(x)\right)=\left(\xi^{\prime}(x) V_{\xi}^{\prime}(x), \xi^{\prime \dagger}(x) V_{\xi}^{\prime}(x)\right) .
$$

This equality is satisfied if and only if

$$
\xi^{\prime}=\xi h, \quad h \xi h=\xi, \quad h \in S U(2) .
$$

It is straightforward to solve this equation for $h \in S U(2)$. Denote the general $S U(2)$ matrix $\xi$ by

$$
\xi=\alpha+i \vec{\beta} \cdot \vec{\tau}, \quad \alpha^{2}+|\vec{\beta}|^{2}=1
$$

If $\alpha \neq 0$, then $\xi$ and $-\xi$ are equivalent. However, if $\alpha=0$, all configurations of the form $i \vec{\beta} \cdot \vec{\tau}$ are equivalent, irrespective of the value of $\vec{\beta}$. If the vector $(\alpha, \vec{\beta})$ 
is considered to be a point on the three sphere $S^{3}$, then we see that points on the northern and southern hemisphere of $S^{3}$ obtained by inversion through the origin are equivalent, and the entire equator is identified to a point. (The resulting manifold is still equivalent to $S^{3}$.) Thus the $\xi$ basis gives a coordinate system for the Goldstone bosons which is well-defined except near the equator of $S^{3}, \xi=i \vec{\beta} \cdot \vec{\tau}$. In the $\Sigma$ basis, the points on the entire three sphere $S^{3}$ correspond to inequivalent field configurations, and the $\Sigma$ basis gives a well-defined coordinate system on all of $S^{3}$.

The chiral Lagrangian for heavy mesons is easy to construct in the $\xi$ basis since the heavy meson fields transform in a simple way under parity. Heavy quark symmetry is incorporated into the chiral Lagrangian by defining a heavy meson field which contains both pseudoscalar and vector mesons. The heavy meson field for the ground state $Q \bar{q}_{a}$ mesons is written as a $4 \times 4$ matrix $^{13}$

$$
H_{a}=\frac{(1+\psi)}{2}\left[P_{a \mu}^{*} \gamma^{\mu}-P_{a} \gamma_{5}\right],
$$

where $v^{\mu}$ is the heavy quark four-velocity, $v^{2}=1$. We shall work in the rest frame of the heavy mesons where $v^{\mu}=(1, \overrightarrow{0})$. The fields $P_{a}, P_{a \mu}^{*}$ destroy the heavy pseudoscalar and vector particles that comprise the ground state $s_{\ell}=1 / 2$ doublets. The vector meson field is constrained by $v^{\mu} P_{a \mu}^{*}=0$. Under $S U(2)_{Q}$ heavy quark spin symmetry

$$
H_{a} \rightarrow S H_{a},
$$

where $S \in S U(2)_{Q}$, and under chiral $S U(2)_{L} \times S U(2)_{R}$

$$
H_{a} \rightarrow H_{b} U_{b a}^{\dagger} .
$$

It is also convenient to introduce

$$
\bar{H}_{a}=\gamma^{0} H_{a}^{\dagger} \gamma^{0}=\left[P_{a \mu}^{* \dagger} \gamma^{\mu}+P_{a}^{\dagger} \gamma_{5}\right] \frac{(1+\psi)}{2}
$$


Under parity

$$
H\left(x^{0}, \vec{x}\right) \rightarrow \gamma^{0} H\left(x^{0},-\vec{x}\right) \gamma^{0}
$$

and

$$
\xi\left(x^{0}, \vec{x}\right) \rightarrow \xi^{\dagger}\left(x^{0},-\vec{x}\right)
$$

The chiral Lagrangian density for heavy meson-pion interactions is ${ }^{14,15,16}$

$$
\begin{aligned}
\mathcal{L}=- & i \operatorname{Tr} \bar{H}_{a} v_{\mu} \partial^{\mu} H_{a}+\frac{i}{2} \operatorname{Tr} \bar{H}_{a} H_{b} v^{\mu}\left[\xi^{\dagger} \partial_{\mu} \xi+\xi \partial_{\mu} \xi^{\dagger}\right]_{b a} \\
+ & \frac{i g}{2} \operatorname{Tr} \bar{H}_{a} H_{b} \gamma^{\nu} \gamma_{5}\left[\xi^{\dagger} \partial_{\nu} \xi-\xi \partial_{\nu} \xi^{\dagger}\right]_{b a}+\ldots,
\end{aligned}
$$

where the ellipsis denotes terms with more derivatives and repeated $a, b$ indices are summed over 1,2. Eq. (22) is the most general Lagrangian density invariant under chiral $S U(2)_{L} \times S U(2)_{R}$, heavy quark spin symmetry and parity. It is easy to generalize this Lagrangian density to include explicit $S U(2)_{L} \times S U(2)_{R}$ symmetry breaking from $u$ and $d$ quark masses and explicit $S U(2)_{Q}$ symmetry breaking from $\Lambda_{Q C D} / m_{Q}$ effects.

The coupling $g$ determines the $D^{*} \rightarrow D \pi$ decay width,

$$
\Gamma\left(D^{*+} \rightarrow D^{0} \pi^{+}\right)=\frac{1}{6 \pi} \frac{g^{2}}{f^{2}}\left|\vec{p}_{\pi}\right|^{3}
$$

The present experimental limit, ${ }^{17} \Gamma\left(D^{*+} \rightarrow D^{0} \pi^{+}\right) \lesssim 72 \mathrm{keV}$, implies that $g^{2} \lesssim 0.4$. In this work the sign of $g$ plays an important role. Applying the Noether procedure to the Lagrangian density in eq. (22) and to the QCD Lagrangian density gives

$$
\bar{q}_{a} \gamma_{\mu} \gamma_{5} T_{a b} q_{b}=-g \operatorname{Tr} \bar{H}_{a} H_{b} \gamma_{\mu} \gamma_{5} T_{b a}+\ldots
$$

where $T$ is a traceless $2 \times 2$ matrix and the ellipsis denotes terms containing the pion fields. Treating the quark fields in eq. (24) as constituent quarks and using the 
nonrelativistic quark model to evaluate the $D^{*}$ matrix element of the l.h.s. of eq. (24) gives $^{16} g=1$. (A similar estimate for the pion nucleon coupling gives $g_{A}=5 / 3$.) In the chiral quark model ${ }^{18}$ there is a constituent-quark pion coupling. Using the chiral quark model gives $g \simeq 0.75$.

The soliton solution of the $S U(2)_{L} \times S U(2)_{R}$ chiral Lagrangian for pion self interactions is

$$
\Sigma=A(t) \Sigma_{0}(\vec{x}) A^{-1}(t)
$$

where

$$
\Sigma_{0}=\exp (i F(|\vec{x}|) \hat{x} \cdot \vec{\tau})
$$

and $A(t)$ contains the dependence on collective coordinates associated with rotations and isospin transformations of the soliton solution,

$$
A=a_{0}+i \vec{a} \cdot \vec{\tau}
$$

where $a_{0}^{2}+|\vec{a}|^{2}=1$. The soliton has the quantum numbers of a baryon containing light $u$ and $d$ quarks. For solitons with baryon number one, the function $F(|\vec{x}|)$ satisfies $F(0)=-\pi$ and $F(\infty)=0{ }^{\star}$. Its detailed shape depends on higher derivative terms in the chiral Lagrangian for pion self interactions. Since $F$ increases as $|\vec{x}|$ goes from zero to infinity, it is expected that $F^{\prime}(0)$ is positive. The baryons containing $u$ and $d$ quarks have wavefunctions that are functions of the $a_{\mu}$. For the neutron and proton states; $|p \uparrow\rangle=(1 / \pi)\left(a_{1}+i a_{2}\right),|p \downarrow\rangle=-(i / \pi)\left(a_{0}-i a_{3}\right),|n \uparrow\rangle=(i / \pi)\left(a_{0}+i a_{3}\right)$ and $|n \downarrow\rangle=$ $-(1 / \pi)\left(a_{1}-i a_{2}\right)$. The soliton $\Sigma_{0}(x)$ is a winding number one map from spacetime into the Goldstone boson manifold. In the $\xi$ basis, the soliton solution has the form (up to an overall sign)

$$
\xi_{0}=\exp (i F(|\vec{x}|) \hat{x} \cdot \vec{\tau} / 2)
$$

As $F$ varies from $F=0$ at spatial infinity to $F=-\pi$ at the origin, $\xi_{0}$ varies from $\xi_{0}=1$, the north pole of $S^{3}$, to $\xi_{0}=-i \vec{\tau} \cdot \hat{x}$, the equator, with intermediate

\footnotetext{
* There appears to be a sign error in the formula for the baryon number current used in Ref. 5 .
} 
points covering the northern hemisphere of $S^{3}$. However, we have already seen that the Goldstone boson manifold in the $\xi$ basis is the northern hemisphere of $S^{3}$, with the equator identified to a point. Thus the soliton solution eq. (28) is a winding number one map. It looks singular at $|\vec{x}|=0$ because the $\xi$ coordinate system for the Goldstone boson manifold is singular at the equator of $S^{3}$. However, this singularity is a reflection of the coordinate singularity on the Goldstone boson manifold in the $\xi$ basis and is not a physically singular field configuration.

The form of the Lagrangian density for heavy meson-pion interactions in eq. (22) is not very convenient for discussing heavy meson-soliton interactions because of the coordinate singularity in $\xi$. It is convenient when dealing with matter fields interacting with solitons to redefine the fields so that the Lagrangian density is expressible in terms of $\Sigma$. For example, introducing new heavy meson fields $P_{a}^{\prime}$ and $P_{a \mu}^{\prime *}$, defined by

$$
H_{a}^{\prime}=H_{b} \xi_{b a}
$$

the $S U(2)_{L} \times S U(2)_{R}$ transformation law in eq. (18) becomes

$$
H_{a}^{\prime} \rightarrow H_{b}^{\prime} R_{b a}^{\dagger}
$$

and the Lagrangian density in eq. (22) becomes

$$
\begin{aligned}
\mathcal{L}=- & i \operatorname{Tr} \bar{H}_{a}^{\prime} v_{\mu} \partial^{\mu} H_{a}^{\prime}+\frac{i}{2} \operatorname{Tr} \bar{H}_{a}^{\prime} H_{b}^{\prime} v^{\mu}\left(\Sigma^{\dagger} \partial_{\mu} \Sigma\right)_{b a} \\
+ & \frac{i g}{2} \operatorname{Tr} \bar{H}_{a}^{\prime} H_{b}^{\prime} \gamma^{\nu} \gamma^{5}\left(\Sigma^{\dagger} \partial_{\nu} \Sigma\right)_{b a}+\ldots
\end{aligned}
$$

The parity transformation rule for the primed heavy meson fields is a little more complicated,

$$
H_{a}^{\prime}\left(x^{0}, \vec{x}\right) \rightarrow \gamma^{0} H_{b}^{\prime}\left(x^{0},-\vec{x}\right) \gamma^{0} \Sigma_{b a}^{\dagger}\left(x^{0},-\vec{x}\right)
$$

Note that in a background Goldstone boson field configuration of a soliton located at the same spatial point as the heavy meson the factor of $\Sigma^{\dagger}$ becomes -1 , whereas 
$\Sigma^{\dagger}=1$ for a heavy meson infinitely far from the soliton. This relative minus sign is the source of the parity flip that gives positive parity heavy meson-soliton bound states.

In the large $N_{c}$ limit, the baryon solitons $B$ containing light $u$ and $d$ quarks are very heavy and time derivatives on the pion fields can be neglected. Consequently the interaction Hamiltonian

$$
H_{I}=-\frac{i g}{2} \int d^{3} \vec{x} \operatorname{Tr} \bar{H}_{a}^{\prime} H_{b}^{\prime} \gamma^{j} \gamma_{5}\left[\Sigma^{\dagger} \partial_{j} \Sigma\right]_{b a}+\ldots
$$

with $\Sigma$ given by eq. (25) determines the potential energy of a configuration with a baryon $B$ at the origin and a heavy $P^{\prime}$ or $P^{\prime *}$ meson at position $\vec{x}$. Using eq. (16) and taking the trace in eq. (33) gives

$$
\begin{gathered}
H_{I}=g \int d^{3} \vec{x}\left[P_{a}^{\prime * j \dagger}(\vec{x}) P_{b}^{\prime}(\vec{x})+P_{a}^{\prime \dagger}(\vec{x}) P_{b}^{\prime * j}(\vec{x})+i \epsilon^{i k j} P_{a}^{\prime * i \dagger}(\vec{x}) P_{b}^{\prime * k}(\vec{x})\right] \\
\times\left[A\left\{\frac{x^{j}}{|\vec{x}|} \hat{x} \cdot \tau\left(F^{\prime}-\frac{\sin (2 F)}{2|\vec{x}|}\right)+\frac{\tau^{j}}{2|\vec{x}|} \sin (2 F)+\epsilon^{j m k} x^{k} \tau^{m} \frac{\sin ^{2}(F)}{|\vec{x}|^{2}}\right\} A^{-1}\right]_{b a}+\ldots
\end{gathered}
$$

Assuming that in attractive channels the potential energy is minimized at $\vec{x}=\overrightarrow{0}$ where the heavy meson and baryon soliton are located at the same point, it is eigenvalues of the potential operator at the origin that are needed to determine the spectrum of bound states. Using eq. (34)

$$
\begin{aligned}
V_{I}(\overrightarrow{0}) & =g F^{\prime}(0)\left[P_{a}^{\prime * j \dagger} P_{b}^{\prime}+P_{a}^{\prime \dagger} P_{b}^{\prime * j}+i \epsilon^{i k j} P_{a}^{\prime * i \dagger} P_{b}^{\prime * k}\right] \\
& \times\left[\left(a_{0}+i \vec{a} \cdot \vec{\tau}\right) \tau^{j}\left(a_{0}-i \vec{a} \cdot \vec{\tau}\right)\right]_{b a}+\ldots
\end{aligned}
$$

where the ellipsis denotes the contribution of terms in the chiral Lagrangian with more than one derivative. It is easy to diagonalize the potential (at the origin) matrix that 
arises from taking the truncated basis of nucleon-heavy meson product states (i.e., $|p \uparrow\rangle\left|P_{1}^{\prime}\right\rangle$, etc.). On this space it is straightforward to show that ${ }^{\star}$

$$
V_{I}(\overrightarrow{0})=-\frac{2}{3} g F^{\prime}(0)\left(\vec{S}_{\ell}^{2}-3 / 2\right)\left(\vec{I}^{2}-3 / 2\right)+\ldots
$$

where $\vec{S}_{\ell}$ denotes the total angular momentum vector of the light degrees of freedom (soliton and heavy meson combined) and $\vec{I}$ denotes the total isospin vector. The eigenstates of $V_{I}(\overrightarrow{0})$ have definite isospin, spin and angular momentum for the light degrees of freedom. They are denoted by $\left|I, s, s_{\ell}\right\rangle$, where $I$ is the total isospin, $s$ the total spin and $s_{\ell}$ is the angular momentum of the light degrees of freedom.

The spatial wavefunctions of the eigenstates of eq. (36) are $\delta^{3}(\vec{x})$, and are parity even. The parity of the meson-soliton bound state is also even, because the primed heavy meson fields are odd under parity at infinity, and so are even under parity at the origin, as noted below eq. (32). The unprimed heavy meson fields have a simple transformation law under parity, and do not have a relative minus sign between the parity at infinity and parity at the origin. However, in the $\xi$ basis, the wavefunction of the bound state contains a factor of the form $\vec{\tau} \cdot \hat{x}$ near the origin. Thus the parity of the soliton is also even in the $\xi$ basis because the negative parity of the spatial wavefunction is combined with the negative intrinsic parity of the heavy meson field. The factor $\vec{\tau} \cdot \hat{x}$ appears singular at the origin, but that is because of the coordinate singularity in the $\xi$ basis. Any physical quantity is well-defined at the origin.

It is interesting to see if the interaction in eq. (33) gives a reasonable spectrum of baryons containing a heavy quark when the effects of operators with more than one derivative are neglected. The first column of energies in Table 1 gives the eigenstates and eigenvalues of $V_{I}(\overrightarrow{0})$ in the truncated basis. Only the $|0,1 / 2,0\rangle,|1,1 / 2,1\rangle$ and $|1,3 / 2,1\rangle$ states are bound if $g$ is taken positive. For the case $Q=c$ these states have the right $I, s, s_{\ell}$ and parity quantum numbers to be the $\Lambda_{c}, \Sigma_{c}$ and $\Sigma_{c}^{*}$ respectively.

\footnotetext{
$\star$ More correctly this gives the matrix elements of the potential operator in eq. (35) divided by the normalization of states. The conventions for the field operators and state normalizations are the same as those in Ref. [14].
} 
In the large $N_{c}$ limit, the $N$ and $\Delta$ are degenerate and the space of states should be enlarged to include products of $\Delta$-baryons with the $P^{\prime}$ and $P^{*^{\prime}}$ mesons. Such states can have the same quantum numbers as the $\Sigma_{c}$ and the $\Sigma_{c}^{*}$. Because the spinspin interaction is suppressed by $1 / N_{c}$, including the $\Delta$ causes the $\Sigma_{c}$ and $\Sigma_{c}^{*}$ to be degenerate with the $\Lambda_{c}$ and gives the energies presented in the last column of Table 1. ${ }^{19}$ The real world is intermediate between the $N_{c} \rightarrow \infty$ limit with a degenerate $N$ and $\Delta$, and the limit where the $\Delta$ is neglected because it is considered to be much heavier than the nucleon. Including the $\Delta$ with a finite $\Delta-N$ mass difference will lead to a $\Sigma_{c}$ state which is heavier than the $\Lambda_{c}$ with a binding energy that is intermediate between the values in the two columns of Table 1. Thus the interaction in eq. (33) gives a reasonable qualitative description of heavy baryons. However it is important to remember that anti-baryon-meson bound states are described by taking $F \rightarrow-F$ so the interaction in eq. (33) gives rise to exotic states. These states can be removed by including the effects of higher derivative terms in the chiral lagrangian. This is not neccessarily in conflict with the derivative expansion, since the normal state binding energy is three times the exotic state binding energy.

In the $N_{c} \rightarrow \infty$ and $m_{Q} \rightarrow \infty$ limit, the most general potential at the origin including all possible higher dimension operators has the form

$$
V=V_{0}(F)-\frac{2}{3} V_{1}(F)\left(\vec{S}_{\ell}^{2}-3 / 2\right)\left(\vec{I}^{2}-3 / 2\right),
$$

on the truncated meson-nucleon subspace. In eq. (39) $V_{0}$ and $V_{1}$ are functionals of the soliton shape function $F(|\vec{x}|)$. The coefficients $V_{0}$ and $V_{1}$ have no definite symmetry under $F \rightarrow-F$, so that the meson-nucleon and meson-antinucleon scattering potentials are not related to each other.

The work presented in this paper is similar in spirit to that of Callan and Klebanov. ${ }^{10}$ The main difference is that constraints imposed by heavy quark symmetry are taken into account. The bound state approach of Callan and Klebanov cannot be taken over to baryons containing a heavy charm or bottom quark without imposing heavy quark symmetry (e.g., there is no reason to expect eq. (6.2) of Ref. 
[10] to hold). The mechanism for the parity flip presented in this paper is the same as that of Callan and Klebanov. For example, if the kinetic energy of the nucleon is included then the ground state is an $L=0$ partial wave, instead of a Dirac delta function. (Some of the excited negative parity baryons containing a heavy quark appear as bound states with odd orbital angular momentum.) However, since the unprimed and primed heavy meson fields are related (near the origin) by a factor of $\hat{x} \cdot \vec{\tau}$ the $S$-wave primed heavy meson-nucleon bound state wave functions get multiplied by a linear combination of $Y_{1, m}$ spherical harmonics in the unprimed basis and hence appear as $P$-waves. Similar changes in quantum numbers also occur in bound states involving monopoles. ${ }^{20}$

Mark B. Wise thanks N. Seiberg for suggesting that it would be interesting to examine how baryons containing a heavy quark arise as solitons.

Table 1

\begin{tabular}{|c|c|c|}
\hline States & Energies neglecting the $\Delta$ & Energies including the $\Delta$ \\
\hline$|0,1 / 2,0\rangle$ & $-3 g F^{\prime}(0) / 2$ & $-3 g F^{\prime}(0) / 2$ \\
$|1,3 / 2,1\rangle,|1,1 / 2,1\rangle$ & $-g F^{\prime}(0) / 6$ & $-3 g F^{\prime}(0) / 2$ \\
$|1,1 / 2,0\rangle$ & $g F^{\prime}(0) / 2$ & $g F^{\prime}(0) / 2$ \\
$|0,3 / 2,1\rangle,|0,1 / 2,1\rangle$ & $g F^{\prime}(0) / 2$ & $g F^{\prime}(0) / 2$ \\
\hline
\end{tabular}

$\star$ With a radial wave function that is strongly peaked about $|\vec{x}|=0$. 


\section{References}

1. E. Witten, Nucl. Phys. B160, 57 (1979).

2. T.H.R. Skyrme, Proc. Roy. Soc. A260, 127 (1961).

3. E. Witten, Nucl. Phys. B223, 433 (1983).

4. J. Wess and B. Zumino, Phys. Lett. B37, 95 (1971).

5. G.S. Adkins, C.R. Nappi and E. Witten, Nucl. Phys. B228, 552 (1983).

6. M. Mattis and M.E. Peskin, Phys. Rev. D32, 58 (1985); M. Karliner and M. Mattis, Phys. Rev. D34, 1991, (1986).

7. N. Isgur and M.B. Wise, Phys. Lett. B232, 113 (1989); Phys. Lett. B237, 527 (1990).

8. H. Georgi, Phys. Lett. B240, 447 (1990).

9. N. Isgur and M.B. Wise, Phys. Rev. Lett. 66, 1130 (1991).

10. C.G. Callan and I. Klebanov, Nucl. Phys. B262, 365 (1985), Phys. Lett. B202, 269 (1988).

11. M. Rho, D.O. Riska, and N.N. Scoccola, Phys. Lett. B 251, 597, (1990); Y. Oh, D. Min, M. Rho, and N. Scoccola, Nucl. Phys. A534, 493 (1991); M. Rho, D.O. Riska, and N.N. Scoccola, Z. Phys. A341, 343, (1992).

12. S. Coleman, J. Wess, and B. Zumino, Phys. Rev. 177, 2239 (1969); C. Callan, S. Coleman, J. Wess, and B. Zumino, Phys. Rev. 177, 2247 (1969).

13. A.F. Falk, H. Georgi, B. Grinstein and M.B. Wise, Nucl. Phys. B343, 1 (1990); J.D. Bjorken, SLAC-PUB-5278, invited talk at Les Rencontres de Physique de la Vallee d'Aoste, La Thuile, Italy (1990) unpublished; H. Georgi, HUTP-91A039, lectures delivered at TASI, (1991) unpublished.

14. M. Wise, Phys. Rev. D45, 2118 (1992).

15. G. Burdman and J.F. Donoghue, Phys. Lett. B280, 287 (1992). 
16. Tung-Mow Yan, Hai-Yang Cheng, Chi-Yee Cheung, Gueg-Lin Lin, Y.C. Lin and Hoi-Lai Yu, CLNS 92/1138 (1992) unpublished.

17. The ACCMOR Collaboration (S. Barlag, et al.), Phys. Lett. B278, 480 (1992).

18. H. Georgi, Weak Interactions and Modern Particle Theory, (Benjamin/Cummings, 1984); A. Manohar and H. Georgi, Nucl. Phys. B234, 189 (1984).

19. Z. Guralnik, M. Luke and A.V. Manohar, UCSD/PTH 92-24 (1992).

20. See, for example, S. Coleman, The Magnetic Monopole Fifty Years Later, in The Unity of Fundamental Interactions, ed. by A. Zichichi, (Plenum, New York, 1983). 